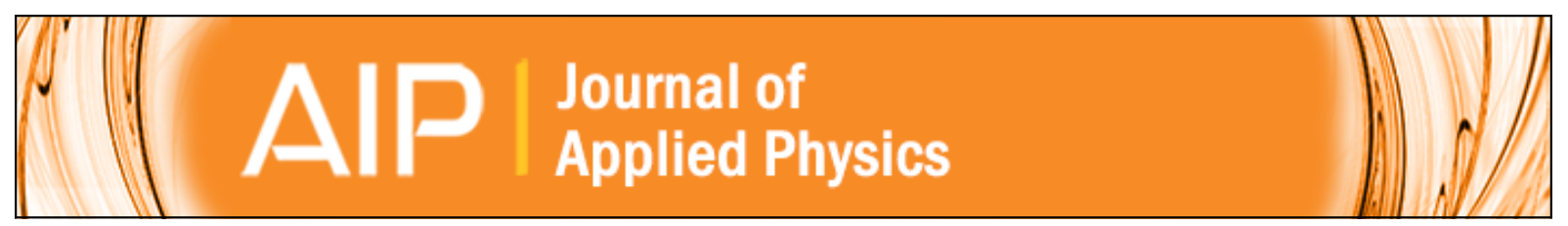

A high-energy electron scattering study of the electronic structure and elemental composition of O-implanted Ta films used for the fabrication of memristor devices

M. Vos, P. L. Grande, S. K. Nandi, D. K. Venkatachalam, and R. G. Elliman

Citation: Journal of Applied Physics 114, 073508 (2013); doi: 10.1063/1.4818524

View online: http://dx.doi.org/10.1063/1.4818524

View Table of Contents: http://scitation.aip.org/content/aip/journal/jap/114/7?ver=pdfcov

Published by the AIP Publishing

Advertisement:

AIP Re-register for Table of Content Alerts 


\title{
A high-energy electron scattering study of the electronic structure and elemental composition of O-implanted Ta films used for the fabrication of memristor devices
}

\author{
M. Vos, ${ }^{1, a)}$ P. L. Grande, ${ }^{2,3}$ S. K. Nandi, ${ }^{2,4,5}$ D. K. Venkatachalam, ${ }^{2}$ and R. G. Elliman ${ }^{2}$ \\ ${ }^{1}$ Atomic and Molecular Physics Laboratories, Research School of Physics and Engineering, \\ The Australian National University, Canberra, ACT 0200, Australia \\ ${ }^{2}$ Department of Electronic Materials Engineering, Research School of Physics and Engineering, \\ The Australian National University, Canberra, ACT 0200, Australia \\ ${ }^{3}$ Instituto de Fisica da Universidade Federal do Rio Grande do Sul, Avenida Bento Goncalves 9500, \\ 91501-970, Porto Alegre, RS, Brazil \\ ${ }^{4}$ Research School of Astronomy and Astrophysics, The Australian National University, Canberra, \\ ACT 2611, Australia \\ ${ }^{5}$ Department of Physics, University of Chittagong, Chittagong, Chittagong - 4331, Bangladesh
}

(Received 22 June 2013; accepted 31 July 2013; published online 20 August 2013)

\begin{abstract}
High-energy electron scattering is used to investigate Ta films implanted with $10 \mathrm{keV} \mathrm{O}$ ions. These films are of interest as they have been used for the fabrication of memristors. High-energy electron scattering is used with incoming electron energies ranging from 5 to $40 \mathrm{keV}$. The inelastic mean free path, and hence the probing depth, is at these energies of the same order as the range of the implanted ions. At the same time, we can distinguish the mass of the atom that scattered the electron elastically, due to the dependence of the recoil energy on the mass of the scatterer. This allows us to determine quantitatively the atomic composition near the surface from the signal of electrons that have scattered elastically but not inelastically. Electrons that have scattered inelastically as well as elastically provide us with information on the possible electronic excitations. Their signal is used to monitor the presence of the $\mathrm{Ta}_{2} \mathrm{O}_{5}$ phase near the surface (characterised by a significant band gap of $\simeq 4.5 \mathrm{eV}$ ), and estimate at what depth below the surface pure Ta metal is present. In this way, we obtain a fairly detailed picture of the elemental composition and electronic properties of these films. (C) 2013 AIP Publishing LLC. [http://dx.doi.org/10.1063/1.4818524]
\end{abstract}

\section{INTRODUCTION}

In nanotechnology, one creates structures near the surface in order to fabricate devices with desired properties. These structures can extend up to depth of $10-40 \mathrm{~nm}$. Measuring the properties of these films are, thus, essential to establish if the structure created has the desired properties. Electron spectroscopic tools such as XPS are very well suited to study the composition and electronic properties of the outermost several nanometers, but are less sensitive to the composition at larger depth. Photoemission based on hard X-rays has been developed to probe deeper below the surface but such experiments can only be done at a few, specialised synchrotron beam lines. Here, we demonstrate an alternative route for the probing of thicker layers, both for composition and electronic structure, based on electron scattering. We use oxygen implanted Ta films as an example, a system that is currently under investigation as a potential candidate for memristor fabrication. ${ }^{1}$ Tantalum oxide films are also widely used in other microelectronic applications. ${ }^{2}$

Memristors based on tantalum oxides rely on the fact that $\mathrm{Ta}_{2} \mathrm{O}_{5}$ has a significant band gap $(\simeq 4.5 \mathrm{eV})$, and hence is highly resistive. Sub-stoichiometric tantalum oxides have no, or much smaller, band gaps and are thus much more

\footnotetext{
a) Author to whom correspondence should be addressed. Electronic mail: maarten.vos@anu.edu.au
}

conductive. For example, the related material and also a potential candidate for memristors, $\mathrm{HfO}_{2}$ is a wide gap insulator, but calculations indicate that $\mathrm{Hf}_{2} \mathrm{O}_{3}$ has a semi-metallic character. ${ }^{3}$ The memristor consists typically of a thin $\mathrm{Ta}_{2} \mathrm{O}_{5}$ layer and an adjacent layer of sub-stoichiometric tantalum oxide. Applying a significant voltage over such a system can result in oxygen (or oxygen-vacancy) migration, and hence the formation of a conductive channel through the $\mathrm{Ta}_{2} \mathrm{O}_{5}$ layer, increasing the conductivity by at least an order of magnitude. Such a conductive channel can be removed by applying another voltage pulse of opposite polarity. In this paper, we investigate the nature of the surface oxide layer formed by $\mathrm{O}$ implantation of Ta as a function of implantation fluence.

For this investigation, we use electron scattering with energies of the incoming electrons $\left(E_{0}\right)$ between $5 \mathrm{keV}$ and $40 \mathrm{keV}$ and electron analyser at a scattering angle $\theta$ of $135^{\circ}$ with respect of the incoming beam. Especially for energies greater than $20 \mathrm{keV}$, the recoil of an electron scattering elastically from an atom is such that it transfers a measurable amount of energy to this atom, an energy that depends on the atom mass $M_{i}$. For scattering from a compound, the elastic peak splits up into different components each corresponding to scattering from an atom with a specific mass. The underlying physics resembles that of (ion) Rutherford Backscattering (RBS) in many ways, and hence we sometimes refer to this technique as electron-RBS or ERBS. The depth that contributes to the elastic peak is determined by 
the path length of the trajectories and the inelastic mean free path $\lambda$. Thus, in this way, electron scattering can determine the composition of the outermost layer. The recoil energy loss $E_{\text {rec }}^{i}$ can be obtained from the well known kinematic factor $K$ and in the electron case it simplifies as the electron mass $m$ is much smaller than the atom mass $M_{i}$

$$
E_{\mathrm{rec}}^{i}=(1-K) E_{0} \approx\left(1+\frac{E_{0}}{2 m c^{2}}\right) \frac{2 m E_{0}}{M_{i}}(1-\cos \theta) .
$$

In contrast to RBS, relativistic corrections have to be taken into account and change the kinematic factor somewhat in these electron scattering experiments. The factor $\left(1+\frac{E_{0}}{2 m c^{2}}\right)$ in the right hand side of Eq. (1) reflects this and it increases the recoil loss at $40 \mathrm{keV}$ by $3.9 \%$. The recoil energy for Ta and $\mathrm{O}$ for $E_{0}=40 \mathrm{keV}$ and $\theta=135^{\circ}$ is $0.43 \mathrm{eV}$ and $4.86 \mathrm{eV}$, respectively, the difference of these recoil energies is easily resolved with a modern spectrometer.

In the above, we implicitly assumed that the target atom was at rest before the collision. In reality it vibrates, even at $0 \mathrm{~K}$ ("zero-point motion"). To see the consequences of this, it is more convenient to analyze the situation in terms of transferred momentum $\mathbf{q}=\mathbf{k}_{0}-\mathbf{k}_{1}$ with $\mathbf{k}_{0}$ and $\mathbf{k}_{1}$ the momentum of the electron before and after the collision, respectively. If the momentum of the scattering atom was $\mathbf{p}$ before the collision, then it changes to $\mathbf{p}+\mathbf{q}$ after the collision. As the recoil energy is equal to the change in kinetic energy of the atom, we can write

$$
E_{\mathrm{rec}}^{i}=\frac{(\mathbf{p}+\mathbf{q})^{2}}{2 M_{i}}-\frac{\mathbf{p}^{2}}{2 M_{i}}=\frac{\mathbf{q}^{2}}{2 M_{i}}+\frac{\mathbf{p} \cdot \mathbf{q}}{M_{i}} .
$$

Thus, the recoil energy has a distribution that is centered around the recoil energy for scattering from a stationary atom (i.e., $\mathbf{q}^{2} / 2 M_{i}$ ) and the shape of the distribution is a Compton profile of the atomic momentum distribution $\mathbf{p}$. In practice, the peak shape is Gaussian and its second moment $\underline{\left(\sigma_{i}\right)}$ can be shown to be related to the mean kinetic energy $\overline{E_{\text {kin }}^{i}}$ of atom $i$ as (see, e.g., Ref. 5) as

$$
\sigma_{i}=\sqrt{\frac{4}{3} E_{\mathrm{rec}}^{i} \overline{E_{\mathrm{kin}}^{i}}} .
$$

In Eq. (2), we make implicitly the assumption that the electron-atom collision can be described as a collision between free particles, i.e., the fact that the atom is bound to a crystal can be neglected. This assumption is far from trivial, but works very well in practice. It is discussed in more detail in, e.g., Ref. 6.

Electrons that scatter inelastically (i.e., create electronic excitations) change their propagation direction only in a very minor way at these high energies and inelastic scattering only will not result in electrons traveling towards the detector. All detected electrons have thus scattered elastically. When electrons that have scattered both elastically and inelastically are measured then the experiment is referred to as (Reflection) electron energy loss spectroscopy ((R)EELS). When high energies are used REELS probes the electronic structure of a relatively thick layer. Of particular interest is the minimum energy loss that can occur when scattering from an insulator or semiconductor. This minimum energy loss corresponds to the band gap and the onset of the energy loss spectrum is thus a signature of the band gap in the near surface area.

The surface sensitivity decreases with increasing values of $E_{0}$. Of course one can increase the surface sensitivity by choosing a more glancing geometry. Another thing worth noting is the difference in depth probed by the elastic signal (no inelastic losses) and the EELS part of the spectrum. For a total path length $l$ the probability $p_{n}(l)$ that there are exactly $n$ inelastic losses is given by a Poisson distribution (see, e.g., Ref. 7)

$$
p_{n}(l)=\left(\frac{l}{\lambda}\right)^{n} \frac{e^{-l / \lambda}}{n !}
$$

with $\lambda$ the inelastic mean free path. Here, we use $\lambda$ values as calculated for $\mathrm{Ta}_{2} \mathrm{O}_{5}$ using the TPP formula. ${ }^{4}$ Examples of these distributions for $n=0$ (elastic peak) and $n=1$ (single inelastic loss event) are given in Fig. 1 for different energies and geometries used in this paper. Clearly, we can probe the sample over a range of thicknesses. For these samples, prepared by $\mathrm{O}$ ion implantation in Ta using an energy of $10 \mathrm{keV}$, the $\mathrm{O}$ concentration is relatively constant from the

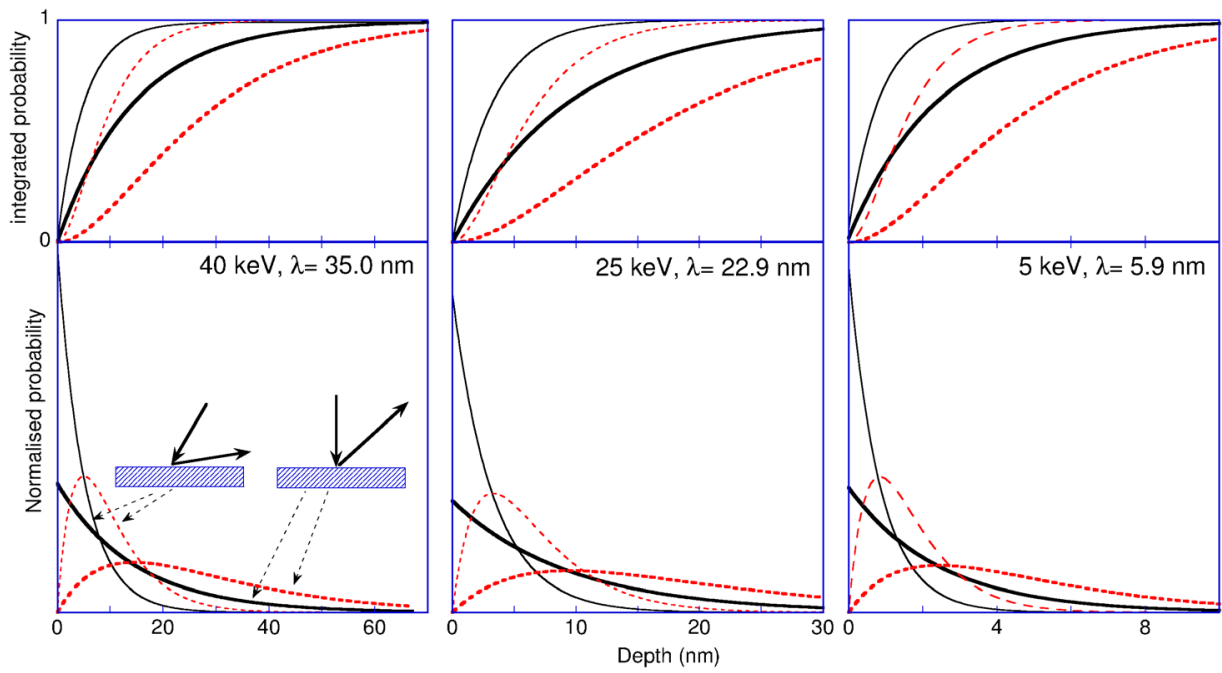

FIG. 1. The lower panels show the depth distribution of elastically scattered electrons without energy loss (solid lines) and elastically scattered electrons with a single inelastic loss event (dashed lines) for incoming energies as shown. Results are given for a measurement geometry with the incoming beam along the surface normal (thick lines) and in a more surface sensitive geometry (rotated by $30^{\circ}$, as indicated in the inset in the $40 \mathrm{keV}$ case). The mean free paths were calculated using the TPP formula for $\mathrm{Ta}_{2} \mathrm{O}_{5} .{ }^{4}$ The upper panels show the integrated curves, i.e., the fraction of scattering events that occurs at a smaller depth. 
surface up to $20 \mathrm{~nm}$ and approaches zero at $40 \mathrm{~nm} .{ }^{8}$ Thus, especially for the loss part of the spectrum, we can probe significantly deeper than the implantation range.

\section{EXPERIMENTAL DETAILS}

$50 \mathrm{~nm}$ thick Ta films were sputter-deposited on a $\mathrm{Si}$ wafer that was capped with a $200 \mathrm{~nm}$ thick thermally grown $\mathrm{SiO}_{2}$ layer. These Ta layers were implanted with $10 \mathrm{keV} \mathrm{O}^{+}$ ions with a fluence between $1 \times 10^{17} / \mathrm{cm}^{2}$ and $5 \times 10^{17} / \mathrm{cm}^{2}$. The samples were characterised by TEM and RBS and electrical measurements ${ }^{8}$ and their working as a memristor device was described in detail as well. ${ }^{9}$ These samples were inserted in the vacuum chamber and measured without further processing. Additionally, we studied $0.1 \mathrm{~mm}$ thick Ta samples that were either sputter-cleaned by $\mathrm{Ar}^{+}$ion etching or on which a $50 \mathrm{~nm}$ thick $\mathrm{Ta}_{2} \mathrm{O}_{5}$ layer was grown by thermal oxidation $\left(600^{\circ} \mathrm{C}\right.$ for $30 \mathrm{~min}$ under a 100 standard cubic centimeters per minute $\mathrm{O}_{2}$ flow). We also studied a $25 \mathrm{~nm}$ thick Ta film deposited on a $\mathrm{Si} / \mathrm{SiO}_{2}$ substrate.

The spectrometer is described in detail elsewhere, see, e.g., Refs. 10 and 11, however, nowadays the detector is read out using a camera, ${ }^{12}$ rather than a resistive anode. Here, we summarize some main points relevant for this work. A small thermal spread electron beam is obtained using a $\mathrm{BaO}$ cathode in the electron gun. Slit lenses are used to focus and decelerate the electron beam from the scattering energy to the pass energy of (nominal) $200 \mathrm{eV}$. A $0.2 \mathrm{~mm}$ wide conical slit aperture placed $130 \mathrm{~mm}$ away from the sample determines which electron enters the analyzer. This means that the scattering angle is well determined (within $0.1^{\circ}$ ) and it is thus possible to explore geometries, where the outgoing trajectory is extremely glancing with the surface. In the current work, we restrict ourselves to outgoing trajectories that are less than $80^{\circ}$ away from the surface normal. The energy resolution of the system is close to $0.3 \mathrm{eV}$ full-width at half maximum.

\section{RESULTS}

\section{A. Reference samples}

Let us first consider some well-defined samples, before considering the ion-implanted samples. In Fig. 2, we show the spectra of electrons reflected from the sample with only little energy loss. The sample was a $50 \mathrm{~nm}$ thick thermally grown tantalum oxide film and the incoming energy $E_{0}$ was $40 \mathrm{keV}, 25 \mathrm{keV}$, and $5 \mathrm{keV}$. Such a thermally grown film is known to be accurately described by the stoichiometry of $\mathrm{Ta}_{2} \mathrm{O}_{5}{ }^{13}$ The main elastic peak is aligned here with zero energy loss. As we will see, this corresponds to electrons scattered from Ta atoms. In the $40 \mathrm{keV}$ and $25 \mathrm{keV}$ spectra, there is a second, much weaker peak, separated from the main peak by $4.4 \mathrm{eV}$ and $2.7 \mathrm{eV}$, respectively. Then, there is an onset of a continuous broad distribution near $4.5 \mathrm{eV}$. We interpret the weaker peaks to be due to electrons scattered from $\mathrm{O}$ atoms, an interpretation that is supported by the fact that the separation from the main elastic peak is exactly what is calculated for the Ta-O separation based on Eq. (1). The broad distribution starting near $4.5 \mathrm{eV}$ is due to electrons

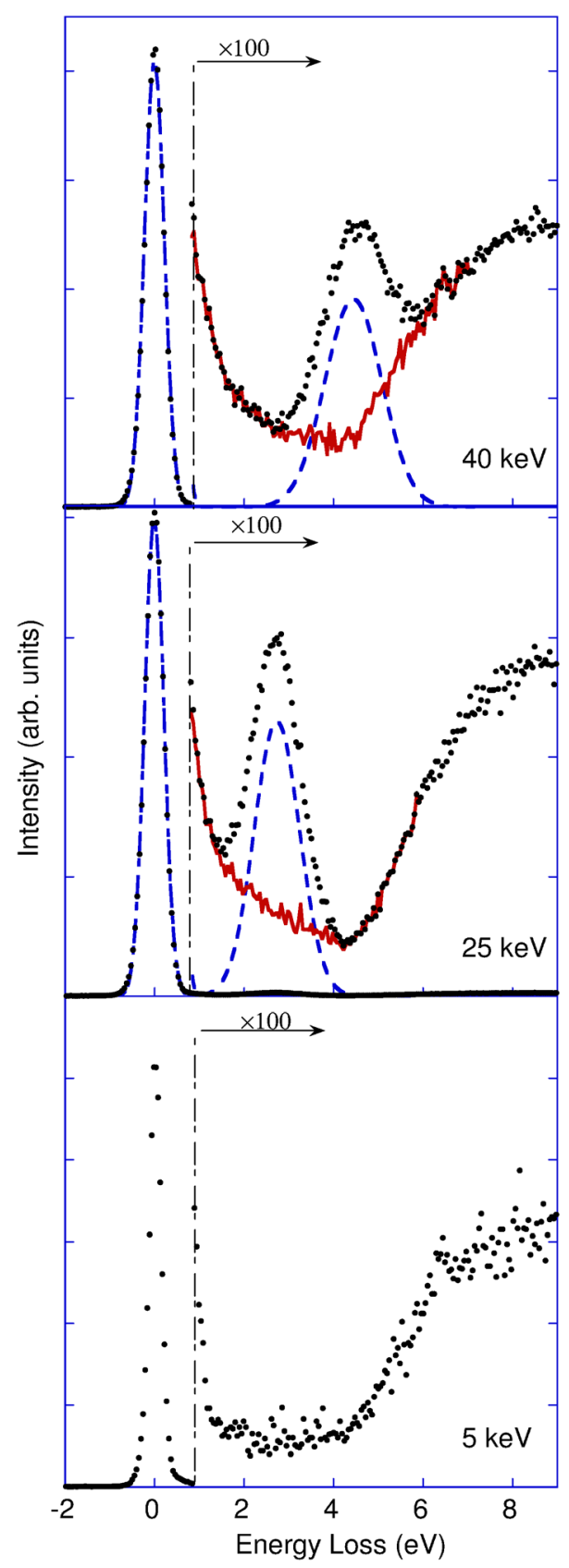

FIG. 2. Spectra of a thick, thermally grown, $\mathrm{Ta}_{2} \mathrm{O}_{5}$ film taken at $40 \mathrm{keV}$, $25 \mathrm{keV}$, and $5 \mathrm{keV}$. In the first two cases, the $\mathrm{O}$ recoil peak is well resolved but the measurement is not background free. The calculated amount of $\mathrm{O}$ elastic peak (based on Ta:O DCS ratio and $\mathrm{Ta}_{2} \mathrm{O}_{5}$ stoichiometry) was subtracted based on a fit of the main Ta and assuming a peak (blue dashed line). The resulting background (red line) is similar in shape to that observed at $5 \mathrm{keV}$ and, in this case, the $\mathrm{O}$ elastic peak has merged with the Ta elastic peak.

scattered elastically from $\mathrm{Ta}$ and, in addition, created an electronic excitation. The minimum energy loss for an electronic excitation in $\mathrm{Ta}_{2} \mathrm{O}_{5}$ is set by the band gap for which values are reported around $4.5 \mathrm{eV} .^{2}$

To confirm this interpretation, we calculated the elastic peak contribution to the spectra assuming a $\mathrm{Ta}_{2} \mathrm{O}_{5}$ stoichiometry. The differential cross sections (DCS) for scattering were obtained from the ELSEPA package. ${ }^{14}$ The resulting cross sections are given in Table I. The theory is quite well established. Basically, under these high-energy and large 
scattering angle conditions, the DCS is determined by accurately known static atomic potential. The influence of polarization and exchange are negligibly small. Absorption (loss of flux due to inelastic channels) has a marginal influence and inclusion of this effect in the calculation is less well established. In Table I, we show the resulting DCS without and with absorption, the latter calculated using the default values of the program. Difference in the obtained DCS ratio of $\mathrm{O}$ and Ta with and without absorption is $3 \%$ at $25 \mathrm{keV}$ and even less at $40 \mathrm{keV}$. For simplicity, we will neglect the influence of absorption here. The $\mathrm{O}$ cross section is 183-184 times smaller than the Ta cross section, whereas the Rutherford cross section (valid for scattering from a bare nucleus) assumes a $Z^{2}$ dependence, which predicts that the $\mathrm{O}$ cross section is only 83.2 times smaller. As discussed elsewhere, the simple Rutherford cross section tends to underestimate the cross sections of high $Z$ elements under these conditions. ${ }^{15}$

We now calculate the elastic peak contribution to the spectrum, assuming Gaussian line shapes, the calculated cross section and an energy resolution of $0.3 \mathrm{eV}$. It is clear from Fig. 2 that the $\mathrm{O}$ and Ta peak have completely different widths. As even the observed width of the Ta elastic peak $(0.5 \mathrm{eV}$ FWHM) is larger than the experimental resolution Doppler broadening contributes to the widths in both cases. Thus, we have to include the intrinsic width (as given by Eq. (3)) as well in the calculation of the elastic peak shape. Extracting a mean kinetic energy of the Ta atoms is difficult as it depends critically of the energy resolution assumed. Moreover, the nature of Eq. (3) implies that a 5\% difference in the observed intrinsic width results in a $10 \%$ difference in mean kinetic energy. In practice, we do not know the energy resolution of the spectrometer precisely enough to extract a meaningful kinetic energy of Ta.

For the O peak, the intrinsic width is difficult to determine as the peak is clearly superimposed on a non-constant background, but is of the order of $1.4 \mathrm{eV}$ (FWHM) at $E_{0}=25 \mathrm{keV}$ and $1.8 \mathrm{eV}$ for $E_{0}=40 \mathrm{keV}$. It is clearly much wider than the energy resolution, and hence the precise value of the assumed energy resolution does not affect the intrinsic width of the $\mathrm{O}$ peak much. We subtracted the calculated $\mathrm{O}$ elastic peak from the observed spectra, and we get a smooth background if we assume the nominal stoichiometry and a kinetic energy of an oxygen atom of $60 \mathrm{meV}$. This approach fails if we assume an $\mathrm{O}$ kinetic energy of more than $65 \mathrm{meV}$ or less than $55 \mathrm{meV}$. The procedure is illustrated in Fig. 2 for both the $40 \mathrm{keV}$ and the $25 \mathrm{keV}$ measurements. Clearly, the analysis is easier in the $25 \mathrm{keV}$ case, as in the $40 \mathrm{keV}$ case

TABLE I. The Differential elastic scattering cross section (in atomic units) at $135^{\circ}$ as calculated with the ELSEPA package. ${ }^{14}$ Values are shown without absorption and as calculated within ELSEPA with the default parameters.

\begin{tabular}{|c|c|c|c|c|}
\hline \multirow{2}{*}{$\begin{array}{l}E_{0} \\
\mathrm{keV}\end{array}$} & \multicolumn{2}{|c|}{ DCS Ta } & \multicolumn{2}{|c|}{ DCS O } \\
\hline & Excl. abs & Incl abs & Excl. abs & Incl. abs \\
\hline 25 & $1.19 \times 10^{-3}$ & $1.13 \times 10^{-3}$ & $6.51 \times 10^{-6}$ & $6.37 \times 10^{-6}$ \\
\hline 40 & $4.57 \times 10^{-4}$ & $4.40 \times 10^{-4}$ & $2.48 \times 10^{-6}$ & $2.44 \times 10^{-6}$ \\
\hline
\end{tabular}

the background appears of different nature before and after the oxygen peak. Hence in the following, we will base the analysis of the amount of $\mathrm{O}$ present in a sample on the $25 \mathrm{keV}$ measurements.

We also measured a spectrum for $E_{0}=5 \mathrm{keV}$. Here, the recoil energies are so small that the different elements are not resolved. Its loss spectrum shows a clear onset near $4.5 \mathrm{eV}$, which we take as a signature of the band gap. This onset is also seen clearly, after subtracting the $\mathrm{O}$ contribution, in the $25 \mathrm{keV}$ and $40 \mathrm{keV}$ spectra. In general, the $5 \mathrm{keV}$ measurements corroborate the background obtained after subtraction of the $\mathrm{O}$ elastic peak in the higher energy measurements.

We measured spectra over a larger energy loss range for both Ta metal (after sputter cleaning a Ta foil with $\mathrm{Ar}^{+}$ions) and $\mathrm{Ta}_{2} \mathrm{O}_{5}$. These are plotted for future reference in Fig. 3

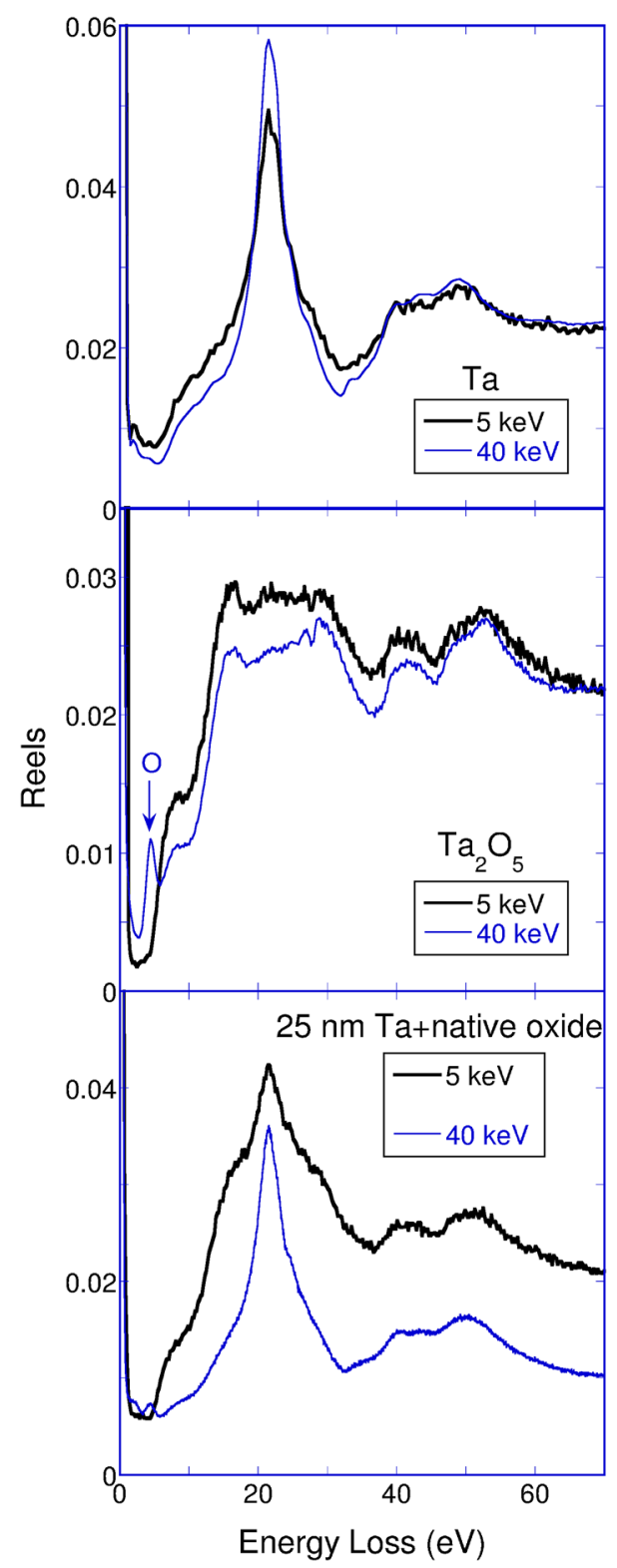

FIG. 3. Energy loss spectra taken at $40 \mathrm{keV}$ and $5 \mathrm{keV}$ for Ta (top) and a $50 \mathrm{~nm}$ thick thermally grown $\mathrm{Ta}_{2} \mathrm{O}_{5}$ layer (middle). The lower panel shows the spectrum of a sputter-deposited and, subsequently, air-exposed Ta film with a native oxide layer. 
with the (Ta) elastic peak normalized to unit area. These measurements were done at $40 \mathrm{keV}$ and $5 \mathrm{keV}$, differences between them are a consequence of different surface plasmon excitation probabilities, as well as different partial intensities (see, e.g., Ref. 16). For Ta metal, the measured spectra resemble the bulk loss function as published by Werner et al. ${ }^{17}$ or the effective loss function at higher energies from Chen et al., ${ }^{18}$ but the present spectra are more intense at larger loss values, as they have not been deconvoluted for multiple energy losses. The low loss region is of most importance as here the elastic peak of $\mathrm{O}$, when present will appear, and this part of the spectrum is not affected by multiple losses. There is a very small peak discernible near $1.7 \mathrm{eV}$ energy loss and an even weaker broad feature near $4 \mathrm{eV}$. These features do not change position if we change the incoming energy (hence they are not due to impurities) and are presumably due to electronic excitations. A peak near $1.7 \mathrm{eV}$ has been reported before in the EELS literature. ${ }^{19}$ Indeed in the density functional calculation of the loss function of Ta small peaks are predicted near $2 \mathrm{eV}$ and $4 \mathrm{eV},{ }^{16,20}$ and these features are also visible in the loss function (Im $(-1 / \varepsilon)$ as derived from the X-ray reflectivity and absorptivity measurements. ${ }^{21}$ These loss features are at similar energy losses as the elastic peak of lighter elements, hence they complicate the interpretation of the experiment when only a small concentration of lighter elements is present. For wide band-gap materials, such problems are absent as no electronic excitations can occur for energies smaller than the band gap.

Within the context of this paper, it is important to note that the shape of the loss spectrum can be used to establish if metallic Ta and/or $\mathrm{Ta}_{2} \mathrm{O}_{3}$ contributes to the loss spectrum. For this purpose, we show the loss spectra of $\mathrm{Ta}_{2} \mathrm{O}_{5}$ over a larger energy loss range as well. Its shape is distinctly different from that of Ta metal.

Finally, we show in the lower panel of Fig. 3, a loss spectrum of a $25 \mathrm{~nm}$ thick Ta film after air exposure (i.e., with the native oxide at the surface). In that case, we get at low energy $\left(E_{0}=5 \mathrm{keV}\right)$, a spectrum that resembles the sum of the spectrum of a $\mathrm{Ta}_{2} \mathrm{O}_{5}$ and metallic Ta film, whereas at high energy $\left(E_{0}=40 \mathrm{keV}\right)$ we obtain a spectrum that approaches that of Ta metal. For the native oxide, the $\mathrm{O}$ elastic peak is visible near $4.5 \mathrm{eV}$, much weaker than in $\mathrm{Ta}_{2} \mathrm{O}_{5}$, but still a considerable stronger peak than the tiny peak due to electronic excitation visible in the spectrum of the pure metal around $4 \mathrm{eV}$. From transmission electron microscopy images ${ }^{8}$ we know that the thickness of the native oxide after implantation is $2-3 \mathrm{~nm}$. If $50 \%$ of the signal at $5 \mathrm{keV}$ is due to metallic $\mathrm{Ta}$, then this would correspond to a $\mathrm{Ta}_{2} \mathrm{O}_{5}$ film thickness of at 4-5 nm (see Fig. 1), somewhat thicker than the thickness of the native oxide as observed in the TEM images for the implanted samples.

\section{B. O implanted Ta samples}

We now focus our attention on the $\mathrm{O}$ implanted $\mathrm{Ta}$ samples. Examples of the elastic peak for the lowest implantation fluence $\left(1 \times 10^{17} / \mathrm{cm}^{2}\right.$ and a highest fluence $\left(5 \times 10^{17} / \mathrm{cm}^{2}\right)$ are given in Fig. 4 . We used the $25 \mathrm{keV}$

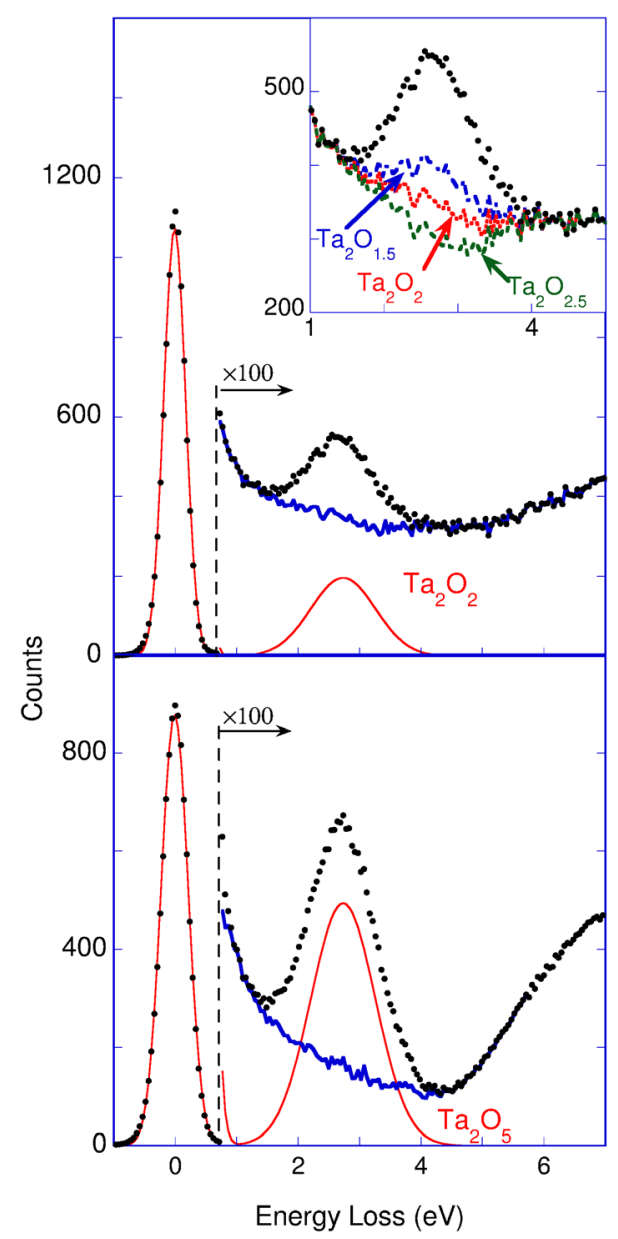

FIG. 4. Spectra (dots) taken at $25 \mathrm{keV}$ for a sample implanted with $1 \times 10^{17} / \mathrm{cm}^{2}$ (top panel) and $5 \times 10^{17} / \mathrm{cm}^{2}$ (lower panel). Ta and $\mathrm{O}$ elastic peaks were simulated for different nominal stoichiometries). The stoichiometry that produced a smooth background after subtracting the $\mathrm{O}$ elastic peak was judged to be the correct one and is shown in the figure as a red (smooth) line. The spectrum after $\mathrm{O}$ peak subtraction is shown as a blue (noisy) line. The variation in the background curves for different choices of stoichiometry is shown as in inset for the $1 \times 10^{17} / \mathrm{cm}^{2}$ sample.

measurement to extract the nominal $\mathrm{O}$ concentration for the two different geometries sketched in the inset of Fig. 1. The $\mathrm{O}$ peak area increases with $\mathrm{O}$ implantation fluence, but not quite proportional, as is clear from Table II. This is attributed to the presence of a native oxide layer, independent of implantation. The measurement with the beam coming in along the surface normal determines the $\mathrm{O}$ concentration averaged over the Ta layer. Here, the contribution of the native oxide at the surface is relatively small. For the measurement after rotation over $35^{\circ}$, (with the outgoing beam quite glancing, $80^{\circ}$ away from the surface normal) the contribution of the native oxide layer is significant. For the lowest implantation fluence, we removed the native oxide layer by sputtering using $2 \mathrm{keV} \mathrm{Ar}^{+}$ions. The sputtering reduced the difference in the $\mathrm{O}$ peak area observed in the bulk- and surface-sensitive geometry (Table II), consistent with a more homogeneous, broad $\mathrm{O}$ distribution in the sample due to implantation. For a fluence of $5 \times 10^{17} / \mathrm{cm}^{2}$, the $\mathrm{O}$ peak area is indistinguishable from that of a thick thermally grown $\mathrm{Ta}_{2} \mathrm{O}_{5}$ film.

These findings are very consistent with sputter-depth profiling of these films in combination with XPS and 
TABLE II. The oxygen concentration (expressed as the $x$ value in $\mathrm{Ta}_{2} \mathrm{O}_{x}$ ) obtained for the $25 \mathrm{keV}$ measurement for the two different measurement geometries. The $1 \times 10^{17}$ implant was measured as received and with the native oxide removed by sputtering.

\begin{tabular}{lcc}
\hline \hline Impl. dose & Perp. in & $35^{\circ}$ rot. \\
\hline $1 \times 10^{17}$ & $2.0 \pm 0.3$ & $3.7 \pm 0.3$ \\
$1 \times 10^{17}$ sp. & $1.1 \pm 0.2$ & $1.5 \pm 0.2$ \\
$2 \times 10^{17}$ & $3.2 \pm 0.3$ & $4.1 \pm 0.3$ \\
$3 \times 10^{17}$ & $4.2 \pm 0.3$ & $5.2 \pm 0.3$ \\
$5 \times 10^{17}$ & $5.1 \pm 0.3$ & $5.2 \pm 0.3$ \\
\hline \hline
\end{tabular}

TEM images, which clearly show the presence of a $2 \mathrm{~nm}$ thick native oxide layer. ${ }^{8}$ XPS also showed that for the $5 \times 10^{17} / \mathrm{cm}^{2}$ implant the $\mathrm{Ta}_{2} \mathrm{O}_{5}$ stoichiometry was reached.

Extending the measurement to larger energy loss ranges gives us, besides the oxygen peak, a fingerprint of the electronic structure. This we show in Fig. 5 for the $40 \mathrm{keV}$ measurement, with the incoming beam directed along the surface normal. Implantation with $1 \times 10^{17} / \mathrm{cm}^{2}$ dampens the main energy loss feature, and this feature decreases further with increasing fluence. Also "shoulders" near $16 \mathrm{eV}$ and $20 \mathrm{eV}$ appear and become gradually more pronounced, and the spectrum starts resembling that of $\mathrm{Ta}_{2} \mathrm{O}_{5}$ but a trace of the Ta metal plasmon peak remains. This is an indication that part of the loss spectrum originates from larger depths than the maximum implantation range of $10 \mathrm{keV} \mathrm{O}$ ions. After high fluence implantation $3-5 \times 10^{17} / \mathrm{cm}^{2}$, the Ta metal related excess intensity near $21 \mathrm{eV}$ energy loss comprises about $10 \%$ of the intensity of the total spectrum. As the Ta layer is originally only $50 \mathrm{~nm}$ thick, such an intensity corresponds to Ta metal being present only in a relatively thin layer $(5-10 \mathrm{~nm})$ close to the $\mathrm{Ta} / \mathrm{SiO}_{2}$ interface. Indeed if the implanted sample is tilted (or the measurement is done at

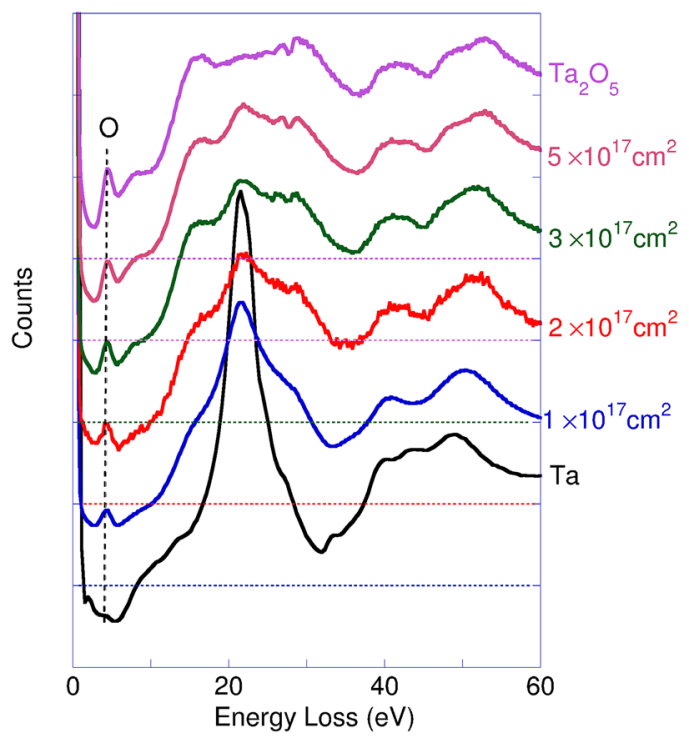

FIG. 5. Energy loss spectra taken at $40 \mathrm{keV}$ for Ta, O implanted Ta samples (implantation dose as indicated) and $\mathrm{Ta}_{2} \mathrm{O}_{5}$. The incoming beam along the surface normal. All elastic peaks are normalized to equal area, but the plots are offset vertically for clarity. The dashed line on the left indicates the position of the oxygen elastic peak. lower incoming energies), so that the measurement becomes more surface sensitive, then the remaining trace of the metallic Ta plasmon disappears, and the spectra after high-fluence implantation become almost identical to that of thick thermally grown $\mathrm{Ta}_{2} \mathrm{O}_{5}$. For $\mathrm{Nb}$ and its various stoichiometric oxides there have been (electron-microscope based) EELS data published. ${ }^{22}$ Unfortunately, this is not the case for Ta and its oxides. The general shape to the $\mathrm{Nb}$ metal loss spectrum and the $\mathrm{Nb}_{2} \mathrm{O}_{5}$ loss spectrum resembles that of Ta metal and thermally grown $\mathrm{Ta}_{2} \mathrm{O}_{5}$, as reported here. The difference in loss function between $\mathrm{Nb}_{2} \mathrm{O}_{5}$ and its other oxides is more subtle. It is thus not clear if these loss spectra contain indications that any other tantalum oxide phases are formed after implantation.

An important part of the EELS is the low-loss range. Here, we can expect to see indications of the minimum excitation energy, which can be related to the band gap. In this range, the interpretation of the loss spectrum is complicated by the presence of the $\mathrm{O}$ elastic peak. In Fig. 6, we show this part of the loss spectrum (for an incoming energy of $25 \mathrm{keV}$ directed along the surface normal) and the background that

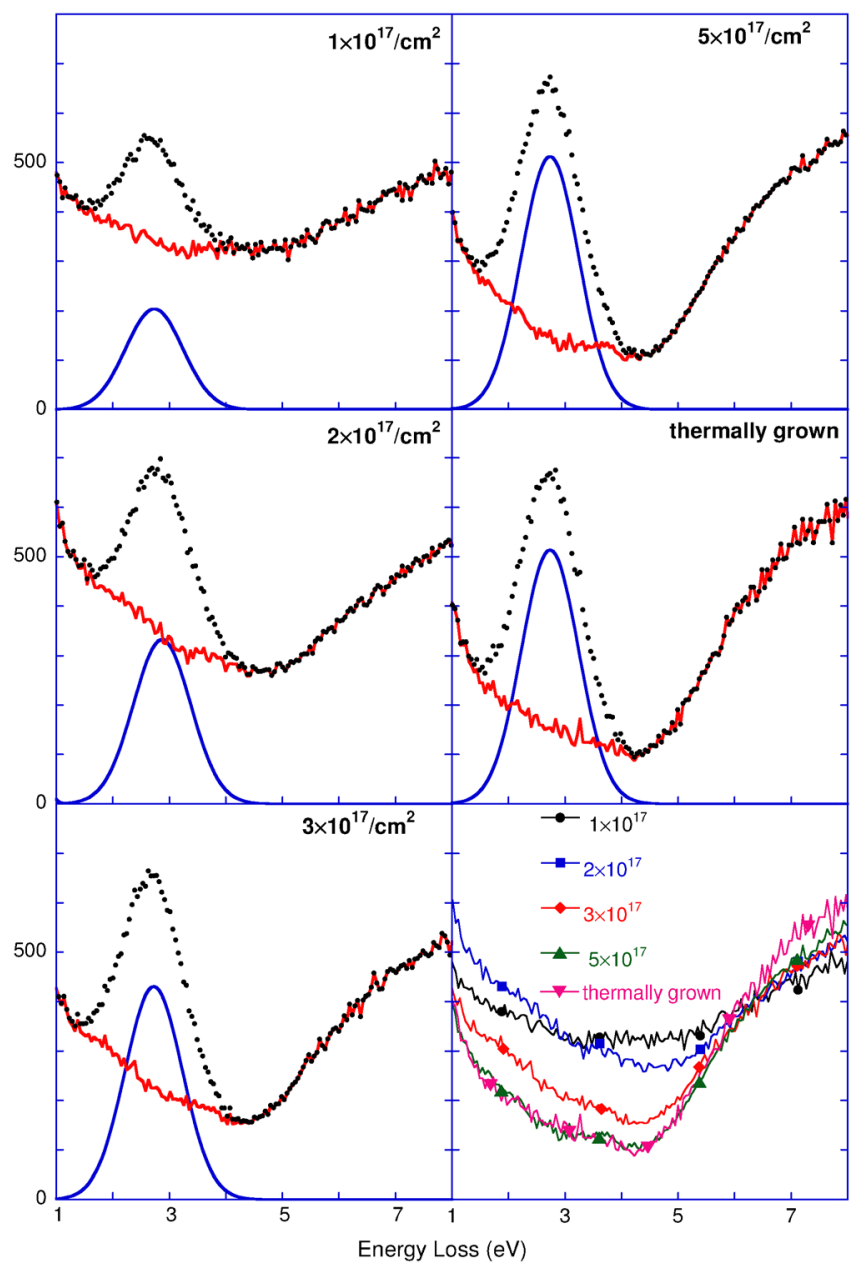

FIG. 6. Energy loss spectra taken (dots) at $25 \mathrm{keV}$ for O implanted Ta samples (implantation fluence as indicated) and thermally grown $\mathrm{Ta}_{2} \mathrm{O}_{5}$. The calculated $\mathrm{O}$ elastic peak contribution (thin line, based on the stoichiometries as shown in Table II) and the background obtained after subtracting this calculated elastic peak from the measured spectrum (thick line) are shown. The lower right panel shows how the background develops as a function of implantation dose. 


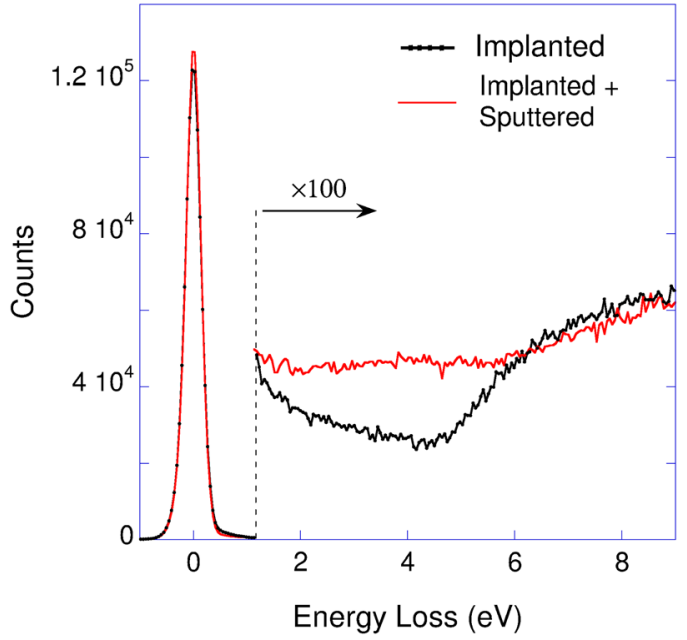

FIG. 7. EELS spectrum, taken at $5 \mathrm{keV}$, of the sample implanted with $10^{17}$ $\mathrm{O}$ ions $/ \mathrm{cm}^{2}$ before and after removal of the native oxide layer by $\mathrm{Ar}^{+}$ sputtering.

is obtained by subtracting the elastic $\mathrm{O}$ peak. The Ta elastic peak of all these spectra was normalized to equal area. Comparing the shape of the loss spectra, we see that with increasing implantation fluence the nature of the background changes gradually. In the low energy loss range $(2-4 \mathrm{eV}$ below the Ta elastic peak), the intensity of the loss spectrum decreases with implantation fluence. Gradually a clear onset of the loss spectrum becomes evident near $4.5 \mathrm{eV}$, and the spectrum starts resembling that of thermally grown $\mathrm{Ta}_{2} \mathrm{O}_{5}$. We interpret this behavior of the loss spectrum as a fingerprint of the band gap developing in the O implanted layer. Note that the position of the onset becomes more pronounced with implantation fluence, but its position does not change. Thus, there is no indication that other phases of $\mathrm{Ta}_{2} \mathrm{O}_{x}$ (than $\mathrm{Ta}_{2} \mathrm{O}_{5}$ ) are present with different band gaps.

For spectra taken in a surface-sensitive geometry (incoming beam rotated by $35^{\circ}$ such that the outgoing trajectories are $80^{\circ}$ away from the surface normal), this onset of the loss spectrum is even visible for the sample implanted with only $1 \times 10^{17} / \mathrm{cm}^{2}$. Here, we see the presence of a native oxide at the surface and the band gap indicates that this layer consists, at least for a large part, of $\mathrm{Ta}_{2} \mathrm{O}_{5}$. After removing the native oxide by in-situ sputtering the onset of the energy loss spectrum disappears for this low fluence sample, even in the surface sensitive geometry. This is illustrated in Fig. 7. Thus, no $\mathrm{Ta}_{2} \mathrm{O}_{5}$ is formed due to implantation of only $1 \times 10^{17} / \mathrm{cm}^{2}$.

\section{CONCLUSION}

We have studied $\mathrm{O}$ implanted tantalum films by highenergy electron scattering and shown that this offers a versatile method of studying the composition and electronic structure of such films. The composition of the films was determined directly from elastically scattered electrons, with the angle and energy dependence of scattering providing additional information about the depth distribution of the implanted oxygen. Additional information about the electronic structure of the films was derived from inelastic electron scattering, including plasmon excitation in Ta metal and band-to-band transitions in ion-beam synthesised $\mathrm{Ta}_{2} \mathrm{O}_{5}$. In the present case, such analysis clearly identified the formation of an insulating $\mathrm{Ta}_{2} \mathrm{O}_{5}$ layer during O-implantation of $\mathrm{Ta}$ at room temperature, and confirmed the presence of a 2-3 nm native $\mathrm{Ta}_{2} \mathrm{O}_{5}$ layer on the Ta surface, both of which are important for resistive switching applications.

\section{ACKNOWLEDGMENTS}

This research was made possible by funding of the Australian Research Council. Oxygen-implanted Tantalum films were provided by Dr. S. Ruffell and Dr. J. England of Varian Semiconductor Equipment, a Division of Applied Materials, as part of a broader collaboration funded by an Australian Research Council Linkage Project Grant. The stay of P.L.G. at the ANU was made possible by a Grant No. 10209/12-3 from CAPES (Brazil). S.K.N. gratefully acknowledges RSAA for his Ph.D. scholarship.

${ }^{1}$ M.-J. Lee, C. B. Lee, D. Lee, S. R. Lee, M. Chang, J. H. Hur, Y.-B. Kim, C.-J. Kim, D. H. Seo, S. Seo, U.-I. Chung, I.-K. Yoo, and K. Kim, Nature Mater. 10, 625 (2011)

${ }^{2}$ C. Chaneliere, J. Autran, R. Devine, and B. Balland, Mater. Sci. Eng. R. 22, 269 (1998).

${ }^{3}$ K.-H. Xue, P. Blaise, L. R. C. Fonseca, and Y. Nishi, Phys. Rev. Lett. 110, 065502 (2013), doi:10.1103/PhysRevLett.110.065502.

${ }^{4}$ S. Tanuma, C. J. Powel, and D. R. Penn, Surf. Interface Anal. 20, 77 (1993).

${ }^{5}$ M. Vos, R. Moreh, and K. Tókési, J. Chem. Phys. 135, 024504 (2011).

${ }^{6}$ M. Vos and M. R. Went, Phys. Rev. B 74, 205407 (2006).

${ }^{7}$ W. S. M. Werner, Surf. Interface Anal. 31, 141 (2001).

${ }^{8}$ S. Ruffell, P. Kurunczi, J. England, Y. Erokhin, J. Hautala, and R. Elliman, Nucl. Instrum. Methods Phys. Res. B 307, 491 (2013).

${ }^{9}$ R. Elliman, M. Saleh, T.-H. Kim, D. Venkatachalam, K. Belay, S. Ruffell, P. Kurunczi, and J. England, Nucl. Instrum. Methods Phys. Res. B 307, 98 (2013).

${ }^{10}$ M. Went and M. Vos, Appl. Phys. Lett. 90, 072104 (2007).

${ }^{11}$ M. Went and M. Vos, Nucl. Instrum. Methods Phys. Res. B 266, 998 (2008).

${ }^{12}$ M. Vos, M. Went, and E. Weigold, Rev. Sci. Instrum. 80, 063302 (2009).

${ }^{13}$ C. A. Steidel, J. Appl. Phys. 40, 3828 (1969).

${ }^{14}$ F. Salvat, A. Jablonski, and C. J. Powell, Comput. Phys. Commun. 165, 157 (2005).

${ }^{15}$ M. Vos, R. McEachran, E. Weigold, and R. Bonham, Nucl. Instrum. Methods Phys. Res. B 300, 62 (2013).

${ }^{16}$ W. Werner, Surf. Sci. 604, 290 (2010).

${ }^{17}$ W. Werner, K. Glantschnig, and C. Ambrosch-Draxl, J. Phys. Chem. Ref. Data 38, 1013 (2009).

${ }^{18}$ T. Chen, Z. Zhang, Z. Ding, R. Shimizu, and K. Goto, J. Electron Spectrosc. Relat. Phenom. 159, 62 (2007).

${ }^{19}$ C. Palacio and J. M. Martinez-Duart, Surf. Interface Anal. 15, 675 (1990).

${ }^{20}$ P. Romaniello, P. de Boeij, F. Carbone, and D. van der Marel, Phys. Rev. B 73, 075115 (2006), doi:10.1103/PhysRevB.73.075115.

${ }^{21}$ J. Weaver, D. Lynch, and C. Olson, Phys. Rev. B 10, 501 (1974).

${ }^{22}$ D. Bach, R. Schneider, D. Gerthsen, J. Verbeeck, and W. Sigle, Microsc. Microanal. 15, 505 (2009). 